\title{
Procédés d'oxydation avancés : utilisation de rayonnement UV dans le domaine de l'environnement
}

J.F. Petrignani

Air Liquide, CRCD, BP. 126, Les Loges, 78350 Jouy-en-Josas, France

Résumé : L'utilisation de rayonnement UV en association avec des oxydants $\left(\mathrm{O}_{2}, \mathrm{H}_{2} \mathrm{O}_{2}, \mathrm{O}_{3}\right)$ permet de générer des espèces radicalaires (HO-) possédant un pouvoir oxydant supérieur aux oxydants de base. L'activation par les UV de photo catalyseurs permet également de produire ces radicaux. Les principes d'activation, les matériels existants et certains développements en cours ainsi que des exemples concrets d'utilisation sont décrits.

\section{INTRODUCTION}

Un des problèmes majeurs pour la sauvegarde de l'environnement est, sans conteste, l'adaptation de solutions techniquement et économiquement viables pour assurer en particulier la qualité des eaux. Pour faire face à une industrialisation et une urbanisation génératrice de pollution, les procédés de traitement chimique par oxydation prennent une place conséquente pour la décoloration, la destruction de matières organiques ou l'amélioration de la biodégradabilité des effluents (1). Parmi les oxydants chimiques utilisables en milieu aqueux, certains sont qualifiès de "propres" du fait que leur réactivité nimplique pas la formation de sous - produits polluants. C'est le cas de l'oxygène, de lozone ou du peroxyde d'hydrogène (2) dont le caractère oxydant peut être quantifié par le potentiel d'oxydoréduction. La figure 1 montre cependant que des espèces radicalaires telles que HO. ont un potentiel supérieur aux oxydants de base et les procédés permettant de les obtenir, appelés procédés d'oxydation avancés sont rassemblés dans la figure 2. Parmi ces procédés, certains sont basés sur l'association d'un oxydant chimique et d'une irradiation ultraviolette(3). 


$\begin{array}{lr}\text { Oxydant } & \begin{array}{c}\text { E, Pot. Ch } \\ (N)\end{array} \\ \text { Radical hydroxyle, } \mathrm{HO} & 2,80 \\ \text { Oxygène atomique, } \mathrm{O} & 2,42 \\ \text { Ozone, } \mathrm{O}_{3} & 2,08 \\ \text { Peroxyde d'hydrogène, } \mathrm{H}_{2} \mathrm{O}_{2} & 1,78 \\ \text { Oxygène, } \mathrm{O}_{2} & 1,23\end{array}$

Figure 1 : Potentiels d'oxydation

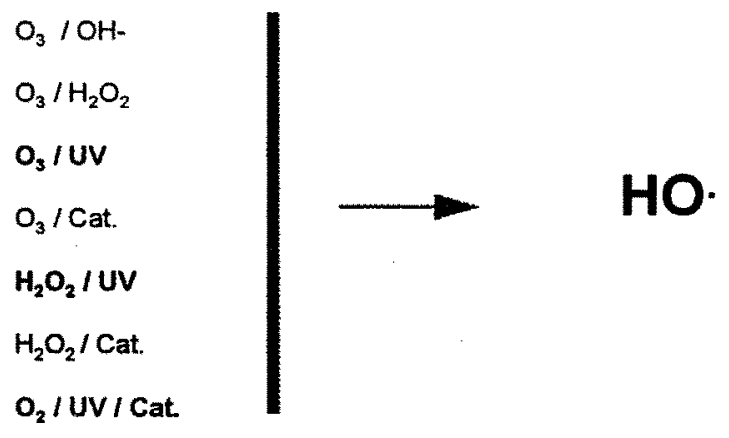

Figure 2 : Procédés d'oxydation avancés

\section{OXYDATION PAR PHOTOLYSE DIRECTE}

Des réactions photochimiques peuvent avoir lieu sur les substrats organiques présents dans l'eau et faciliter leur oxydation. Ces réactions mettent en jeu des transferts électroniques entre un état excité du substrat (eq 1) et le dioxygène (eq 2) suivi de recombinaison ou d'hydrolyse du radical anion ou bien coupure homolytique du composé organique (eq 3) suivi d'une oxydation par $\mathrm{O}_{2}$ (eq 4). Cette situation existe notamment pour les produits halogénés. Les paramètres importants sont le rendement quantique, la quantité de photons émis à la longueur d'onde considérée et la concentration en oxygène dissous. 


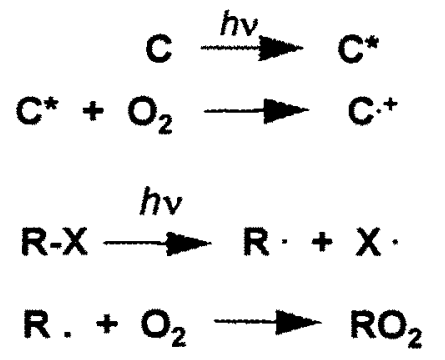

Dans la plupart des cas ces phénomènes sont complémentaires aux réactions photochimiques liées à l'oxydant pour la génération des radicaux, hydroxyle $\mathrm{HO}$.

\section{MECANISMES D'OXYDATION AVANCES}

\section{1. $\mathrm{H}_{2} \mathrm{O}_{2} / \mathrm{UV}$}

Les mécanismes mis en jeu dans cette association sont représentés dans la figure 3 . Les radicaux formés peuvent évoluer par abstraction d'hydrogène, addition électrophile, transfert d'électron ou recombinaison permettant l'obtention de produits organiques oxydés. La réaction d'initiation des radicaux à partir du peroxyde d'hydrogène s'effectue avec un rendement quantique faible. La valeur du coefficient d'extinction du peroxyde est liée au $\mathrm{pH}$ de la solution.

$$
\begin{aligned}
\mathrm{H}_{2} \mathrm{O}_{2} \stackrel{h v}{ } 2 \mathrm{HO} \cdot \Phi=0,98 \quad \varepsilon_{\mathrm{H}_{2 O 2}}: 18,6 \mathrm{M}^{-1} \mathrm{~cm}^{-1}(253,7 \mathrm{~nm}) \\
\varepsilon_{\mathrm{H} 2 \mathrm{O} .}: 240 \mathrm{M}^{-1} \mathrm{~cm}^{-1}(253,7 \mathrm{~nm}) \\
=\quad \text { Influence du pH }
\end{aligned}
$$

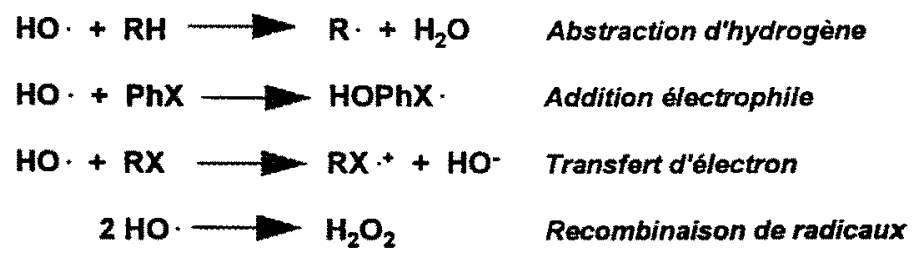

Figure $3: \mathrm{H}_{2} \mathrm{O}_{2} / \mathrm{UV}$; mécanismes d'oxydation.

\section{2. $\mathrm{O}_{3} / \mathrm{UV}$}

Lutilisation de l'ozone dans le traitement des eaux a fait l'objet de nombreuses réalisations industrielles et l'association avec une source UV permet d'augmenter, là encore, le potentiel d'oxydation du système. Différents mécanismes sont proposés. La formation d'oxygène singulet (eq 5) et sa dissociation dans l'eau ne semblent pas cependant être la véritable initiation dans laquelle les radicaux formés ne quitteraient pas

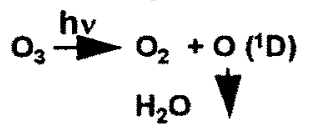


la cage du solvant. Il semble que la première réaction soit la formation de peroxyde d'hydrogène selon une séquence mécanistique possible développée dans la figure 4.

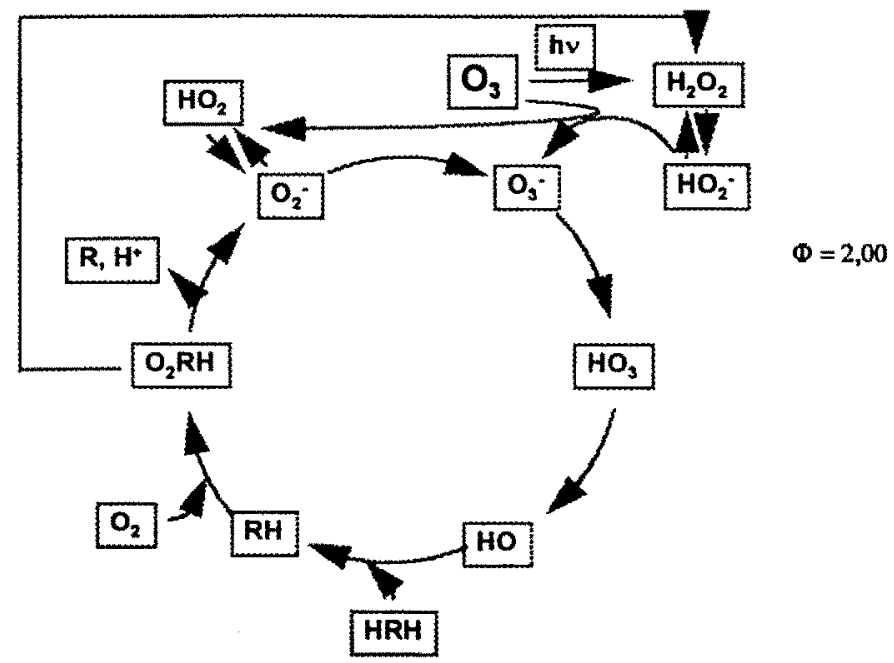

Figure $4: \mathrm{O}_{3} / \mathrm{UV}$; mécanismes réactionnels

\subsection{Oxydation photo catalytique}

Le principe est basé sur la photosensibilité de semi-conducteurs tels que $\mathrm{TiO}_{2}$ (sous la forme rutile). Sous l'action d'un rayonnement (UVA, UVB, UVC), l'électron passe de la bande de valence à la bande de conduction en laissant un trou. A la surface du catalyseur des réactions d'adsorption et de décomposition de l'eau vont conduire à l'obtention de radicaux $\mathrm{HO}$. La présence d'oxygène dissous ou de peroxyde permet de piéger l'électron dans la bande de valence en empêchant la recombinaison avec le trou (cf figure 5). Le catalyseur peut être en suspension dans l'eau ou supporté sur un matériau. Les recherches actuelles portent principalement sur l'élaboration de ces catalyseurs, l'avantage étant l'utilisation potentielle de la lumière solaire.

$$
\mathrm{nO}_{2} \stackrel{\text { hy }}{\mathrm{nO}} \mathrm{NO}_{2}\left(\mathrm{e}^{*}+\mathrm{t}^{+}\right) \quad \Phi=0,05
$$

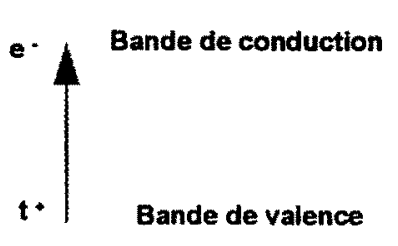

$$
\begin{aligned}
& \begin{array}{l}
\mathrm{MO}_{2}\left(e^{-}\right)+\mathrm{O}_{2} \\
\mathrm{TO}_{2}\left(e^{-}\right)+\mathrm{H}_{2} \mathrm{O}_{2} \longrightarrow \mathrm{TiO}_{2}+\mathrm{O}_{2}^{-} \\
\mathrm{TiO}_{2}+\mathrm{HO}^{-}+\mathrm{HO}
\end{array} \\
& \begin{array}{l}
\mathrm{TO}_{2}(t+1)+\mathrm{RX}_{\text {ads }} \rightarrow-\mathrm{TiO}_{2}+\mathrm{RX}_{2 d t} \\
\mathrm{TiO}_{2}(t)+\mathrm{H}_{2} \mathrm{O}_{\text {dd }} \rightarrow-\mathrm{TiO}_{2}+\mathrm{HO}
\end{array}
\end{aligned}
$$

Figure 5 : Oxydation photo catalytique 


\subsection{UV du vide}

Dans ce domaine spectral $(<190 \mathrm{~nm})$ où l'oxygène de l'air absorbe le rayonnement, la nature de la source (vide ou gaz non absorbants) et la technologie du réacteur (réacteur film) sont prépondérantes. La réactivité est liée à la coupure homolytique de molécules, ce qui, dans le cas de l'eau, permet de générer les radicaux $\mathrm{HO}$.

$$
\mathrm{H}_{2} \mathrm{O} \stackrel{\text { hv }}{\longrightarrow} \mathrm{H} \cdot+\mathrm{HO} \text {. }
$$

Le principal avantage est l'obtention des radicaux sans adjonction d'oxydant chimique. Les applications restent néanmoins limitées à des eaux très peu chargées (élimination de traces dans l'eau ultra pure par exemple). Le développement de cette technique passe par lamélioration du rendement énergétique des sources utilisées.

\section{SOURCES UV}

Les récents développements de ces différents procédés d'oxydation sont, pour une grande part, dus au progrès effectué au niveau des sources UV qui peuvent être classées de la manière suivante :

- Basse pression de vapeur de mercure (254 nm) avec ou sans enveloppe pour filtrer l'émission à $185 \mathrm{~nm}$.

- Moyenne ou haute pression de vapeur de mercure, avec ou sans dopants pour changer la distribution du spectre d'émission.

- Lampe xénon avec ou sans dopants, parfois pulsée pour augmenter l'émission dans les longueurs d'onde $<270 \mathrm{~nm}$.

- Laser eximer ("décharge silencieuse") contenant gaz rares, halogénures de gaz rares ou mẻlanges gaz rares $/ \mathrm{Hg}$.

La connaissance et l'adaptation du spectre d'émission de ces sources en fonction du procédé d'oxydation envisagé et éventuellement de la nature des produits en solution dans l'eau sont fondamentales pour la viabilité économique du procédé. A titre de comparaison, un critère d'efficacité a été récemment proposé reliant la consommation électrique à l'élimination de différents substrats organiques $(4)$. Cette grandeur appelée énergie électrique par ordre de grandeur de polluant détruit s'exprime de la façon suivante :

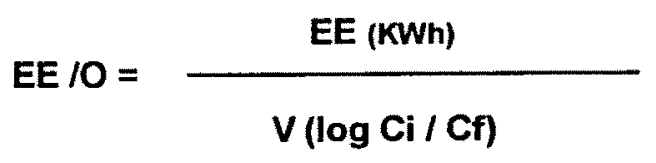

\footnotetext{
EE : Energie électrique nécessaire pour détruire un polluant de la concentration $\mathrm{Ci}$ jusqu'a $\mathrm{Cf}$ dans un volume $V$
}

Cette formule implique que la cinétique de destruction du produit soit d'ordre 1 par rapport à la concentration, ce qui représente le cas le plus communément observé. Le tableau 1 donne des exemples de valeurs de ce critère pour le traitement de $1000 \mathrm{US}$ Gallons par oxydation en utilisant le procédé $\mathrm{H}_{2} \mathrm{O}_{2} / \mathrm{UV}$. 


\section{Tableau 1 : Critère d'efficacité énergétique}

\section{COMPOSE EE/O}

(kWh/1000gal / ordre)

$\begin{array}{ll}\text { 1,4- Dioxane } & 6 \\ \text { Atrazine } & 30 \\ \text { Benzene } & 5 \\ \text { Toluene } & 5 \\ \text { Xylène } & 5 \\ \text { Chlorobenzène } & 5 \\ \text { DCE } & 5 \\ \text { Cyanure de fer } & 40 \\ \text { TNT } & 12 \\ \text { Chlorure de vinyle } & 3 \\ \text { Phénol } & 5 \\ \text { Pentachlorophénol } & 10 \\ \text { Diméthylamine } & 5\end{array}$

\section{Données Solarchem Environmental Systems}

\section{APPLICATIONS}

Ces procédés d'oxydation permettent de résoudre un certain nombre de problèmes inhérents au traitement des effluents aqueux :

- DESINFECTION

- DECOLORATION

- DESODORISATION

- DIMINUTION DU TAUX DE MATIERES OXYDABLES

- AMELIORATION DE LA BIODEGRADABILITE

- ELIMINATION D'ESPECES TOXIQUES

Dans une chaîne classique de traitement d'eau, le positionnement de ce type de procédé est, soit en tant que traitement de finition (après une station d'épuration biologique par exemple), soit en amont, pour améliorer la biodégradabilité de l'effluent avant cette étape biologique.

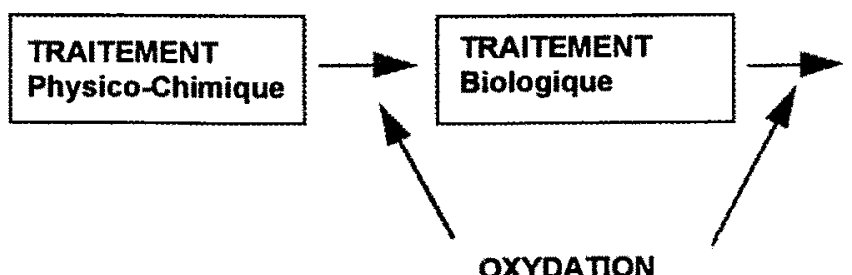

Figure 6 : Positionnement d'un traitement par procédé d'oxydation avancé 
Certains de ces procédés sont déjà appliqués pour traiter des effluents industriels (en particulier aux Etats-Unis) et les Sociétés qui commercialisent ces procédés et matériels mènent ou sont demandeurs $\mathrm{d}$ ' actions en $\mathrm{R} \& \mathrm{D}$ pour améliorer l'efficacité et les coûts $(5)$ en particulier dans le domaine des sources UV.

\section{Références}

[1] Doré M., Chimie des oxydants et traitement des eaux (Technique et documentation Lavoisier, Paris 1989).

[2] Boisselier B, Petrignani JF, Rabergeau G. and Ledon H., L'eau, l'industrie les misances, 166 (1993) 105-109.

[3] Legrini O., Oliveros E., and Braun A.M., Chem. Rev. 93 (1993) 671-698.

[4] Glaze W.H., Ozone News, 22 (1994) 42-46.

[5] Bolton J.R., Cater S. and Safarzedeh-Amiri A., "Advanced Oxidation Technologies for the Photodegradation of Organic Pollutants in Water", Chemical Oxidation Technologies for the Nineteens, Nashville 14-18 Fevrier 1994 (Technomic). 